\title{
Cisto ósseo simples: relato de casos clínicos
}

Simple bone cyst: report of cases

\author{
Erika Barboza Ribeiro \\ Flávia Carneiro Tagliari Bisol** \\ Lilian Cristina Vessoni Iwaki** \\ Mariliani Chicarelli da Silva ${ }^{* * *}$ \\ Elen de Souza Tolentino***
}

\section{Resumo}

Introdução: o Cisto Ósseo Simples (COS) é classificado pela Organização Mundial de Saúde (OMS) como uma lesão não neoplásica relacionada aos ossos, sendo definida como um cisto intraósseo. A causa e a patogênese são incertas e controversas. Grande parte das lesões são cavidades vazias, contendo não mais que um pequeno fluido seroso ou serosanguinolento, estando o revestimento epitelial ausente. Os locais mais acometidos são ossos longos e gnáticos. Os ossos gnáticos surgem com maior frequência na mandíbula em área de molares e pré-molares, com caráter assintomático, porém podem ser relatadas dor e parestesia em alguns casos. Objetivo: este trabalho visa relatar três casos de COS com manifestações radiográficas e condutas terapêuticas distintas. Considerações finais: o diagnóstico do COS é baseado, primariamente, em características clínicas e radiográficas juntamente com os achados cirúrgicos. Radiograficamente apresenta-se como uma lesão radiolúcida, bem delimitada, podendo ser observadas interdigitações entre as raízes dentárias. A forma de tratamento indicada é a curetagem da lesão, com acompanhamento radiográfico pós-cirúrgico para preservação do caso, ou em casos em que as características clínicas e radiográficas são bastante sugestivas, pode-se optar somente pela proservação.

Palavras-chave: Cistos ósseos. Mandíbula. Radiografia panorâmica.

\section{Introdução}

O Cisto Ósseo Simples (COS) é classificado pela Organização Mundial de Saúde (OMS) ${ }^{1}$ como uma lesão não neoplásica relacionada aos ossos, sendo definida como um cisto intraósseo, que apresenta uma cápsula tênue ou um tecido conectivo sem epitélio ${ }^{2}$. O termo "cisto" é geralmente mal aplicado, visto que grande parte das lesões são cavidades vazias, contendo não mais que um pequeno fluido seroso ou serosanguinolento, estando o revestimento epitelial ausente ${ }^{3}$. Apresenta como sinônimos: cisto ósseo solitário, cisto ósseo traumático, cisto ósseo hemorrágico, cisto ósseo unicameral, cisto hemorrágico e cavidade óssea idiopática ${ }^{1}$.

A causa e a patogênese são incertas e controversas, são várias as propostas teóricas, mas nenhuma explicação compatível para todas as características clínicas e patológicas dessa doença. A hipótese "trauma hemorragia" é a mais aceita dentro da literatura, pois sugere que, em um traumatismo ósseo insuficiente para causar fratura, a resultante é um hematoma intraósseo e, se esse não sofrer organização e reparo pode se liquefazer, resultando em um defeito cístico ${ }^{4}$.

\footnotetext{
Especialista em Radiologia Odontológica e Imaginologia pelo Instituto Ravel de Ensino Superior, Maringá, Paraná, PR, Brasil.

Graduanda do Departamento de Odontologia da Universidade Estadual de Maringá (UEM), Maringa, Paraná, PR, Brasil.

** Professora Doutora do Departamento de Odontologia da Universidade Estadual de Maringá (UEM), Maringá, Paraná, PR, Brasil.
} 
Os COS têm sido relatados em quase que a totalidade dos ossos do corpo humano, mas a grande maioria envolve os ossos longos. São comuns nos ossos gnáticos e mais frequentemente encontrados em pacientes entre 10 e 20 anos de idade. A lesão é rara em crianças com menos de 5 anos e pouco frequente em pacientes depois dos 35 anos. Os COS dos ossos gnáticos são, essencialmente, restritos à mandíbula, embora existam relatos da lesão na maxila, e ocasionalmente encontrados bilateralmente na mandíbula. Aproximadamente $60 \%$ dos casos ocorrem em homens ${ }^{5}$.

O COS geralmente não produz sintomas e é descoberto apenas quando as radiografias são feitas por qualquer outra razão. Cerca de $20 \%$ dos pacientes têm aumento de volume indolor da área afetada. Podem ser relatadas dor e parestesia em alguns casos. Embora qualquer área da mandíbula possa estar envolvida, os COS são mais comuns nas áreas de molares e pré-molares ${ }^{6}$.

Radiograficamente, a lesão aparece como um defeito radiolúcido bem delimitado. Em algumas áreas, as margens do defeito são precisamente definidas e, em outras, as margens são mal definidas. O defeito pode variar de $1 \mathrm{a} 10 \mathrm{~cm}$ de diâmetro. Quando vários dentes estão envolvidos na lesão, o defeito radiolúcido frequentemente mostra projeções em forma de cúpula, que se dirigem para cima entre as raízes dos dentes. Essa característica é altamente sugestiva, mas não é diagnóstica de COS. Em muitos casos pode ser notado um contorno em forma de cone (apontando para uma ou ambas as extremidades na direção póstero-anterior) particularmente quando a lesão é grande. Também são possíveis margens ovais, irregulares ou arredondadas. Os dentes que parecem estar envolvidos na lesão são geralmente vitais e não mostram reabsorção radicular ${ }^{5}$. Segundo Harris et al. ${ }^{7}$ (1992), não há evidencias de deslocamento dental e a lâmina dura do dente não é afetada, porém, usualmente aparece atenuada.

As características radiográficas do COS, embora frequentemente sugiram o diagnóstico, não o fecham e podem ser confundidas com uma grande variedade de lesões odontogênicas e não odontogênicas radiolúcidas dos ossos gnáticos. É necessária exploração cirúrgica para estabelecer o diagnósti$\mathrm{co}^{5}$. Exames complementares, como teste de vitalidade pulpar, tomografia computadorizada e biópsia, são importantes para estabelecer a hipótese diagnóstica. O diagnóstico diferencial inclui tumor odontogênico queratocístico, lesão central de células gigantes, ameloblastoma, mixoma e outros cistos e tumores odontogênicos ${ }^{8}$.

Como frequentemente é obtido pouco ou nenhum tecido no momento da cirurgia, o diagnóstico do COS é baseado, primariamente, em características clínicas e radiográficas juntamente com os achados cirúrgicos. Em aproximadamente um terço dos casos, o defeito lítico será observado como sendo uma cavidade vazia com paredes ósseas lisas e brilhantes. Em aproximadamente dois terços dos casos, a cavidade irá conter pequenas quantidades de fluido serossanguinolento. $\mathrm{O}$ feixe neurovascular mandibular pode ser visto, repousando livremente na cavidade ${ }^{5}$.

A forma de tratamento do COS, acordado por diversos autores na literatura ${ }^{9-11}$, é a cirurgia por meio de curetagem rigorosa para estimular o sangramento dentro da cavidade que se encontra vazia. A estimulação do sangramento faz com que a cavidade seja tamponada e que ocorra neoformação óssea com a chegada de células indutoras da osteogênese. Porém, pode-se optar pelo fechamento por segunda intenção, com curativos com gaze furacinada, que deve ser trocada a cada dois dias até o total fechamento da cavidade cirúrgica, além de irrigação da cavidade remanescente com soro fisiológico $0,9 \%$ e bochecho com digluconato de clorexidina $0,12 \%$, três vezes ao dia ${ }^{12}$. A escassez de conhecimento sobre sua natureza e seu comportamento biológico reflete a divergência existente na literatura em relação às formas de tratamento. A raridade de sua ocorrência em adultos sugere uma autolimitação da doença. A resolução espontânea é relatada na literatura, colocando em questionamento a real necessidade de um tratamento cirúrgico ${ }^{13}$.

O objetivo deste trabalho é apresentar e discutir três casos clínicos de COS e compará-los clínica e radiograficamente às características descritas na literatura.

\section{Relato de casos}

\section{Caso clínico 1}

Paciente de 16 anos de idade, gênero feminino, melanoderma. Na radiografia panorâmica, observou-se uma área radiolúcida unilocular, bem delimitada, com halo radiopaco, de aproximadamente 3 $\mathrm{cm}$ de diâmetro, localizado entre os dentes 42 e 45, com projeções em forma de cúpula entre as raízes dos dentes, que não foram reabsorvidas e apresentavam vitalidade pulpar (Figura 1). A lesão era assintomática e não expansiva.

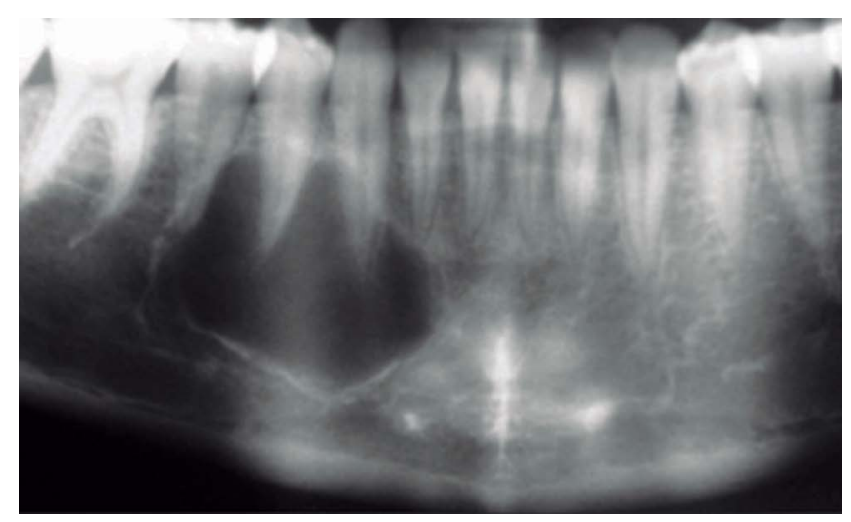

Fonte: dos autores.

Figura 1 - Detalhe da radiografia panorâmica, evidenciando área radiolúcida, a qual apresenta projeções em forma de cúpula 
A hipótese diagnóstica foi de COS e lesão central de células gigantes. Por esse motivo, optou-se por fazer uma exploração cirúrgica da lesão, na qual se observou ausência de revestimento epitelial e a cavidade encontrava-se vazia. Frente às características clínicas, radiográficas e transcirúrgicas, o diagnóstico foi de COS. A forma de tratamento escolhida foi a cirúrgica, por meio de curetagem rigorosa. Após oito anos de proservação, observou-se radiograficamente uma neoformação óssea na região (Figura 2).

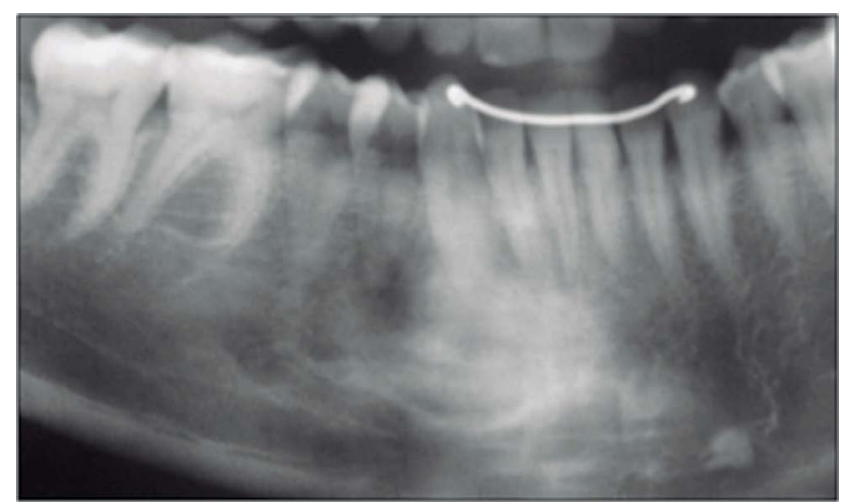

Fonte: dos autores.

Figura 2 - Neoformação óssea da região no pós-operatório de oito anos

\section{Caso clínico 2}

Paciente do gênero masculino, 22 anos de idade, leucoderma, portando uma radiografia panorâmica realizada em outro serviço há um ano, a partir da qual foi notada a presença de uma área radiolúcida unilocular, assintomática do lado esquerdo da mandíbula, com formação de cúpula nas raízes do dente 36 (Figura 3). Foram feitos testes de vitalidade pulpar nos dentes adjacentes e homólogos. Todos apresentaram-se vitais, sem reabsorções radiculares e sem deslocamento. Frente às características clínicas e radiográficas, o diagnóstico presuntivo foi de COS. A conduta tomada foi a realização de controles radiográficos a cada três meses. No acompanhamento de um ano, a radiografia panorâmica revelou estabilização da lesão com aparente início de involução e neoformação óssea. O paciente encontra-se em proservação, com consultas regulares de controle radiográfico.

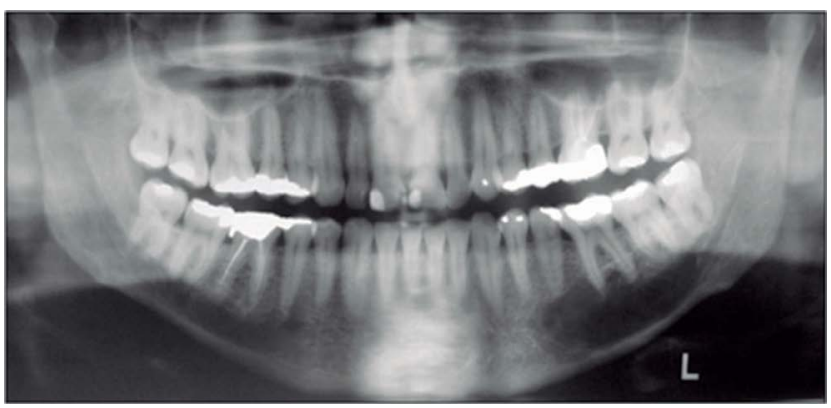

Fonte: dos autores.

Figura 3 - Radiografia panorâmica inicial, evidenciando lesão em corpo de mandíbula esquerdo

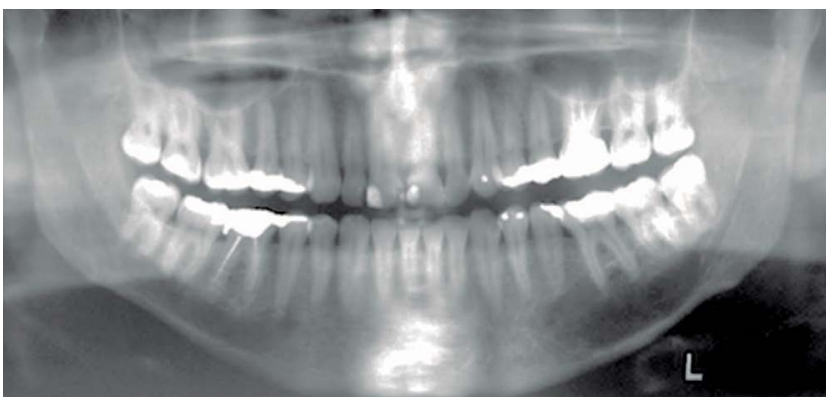

Fonte: dos autores.

Figura 4 - Radiografia panorâmica no acompanhamento de doze meses, demonstrando estabilização da lesão

\section{Caso clínico 3}

Paciente do gênero masculino, com 19 anos de idade, leucoderma, foi encaminhado para exodontia dos terceiros molares. Ao observar o exame radiográfico, foi diagnosticada uma lesão radiolúcida, unilocular, localizada na região do ângulo da mandíbula do lado esquerdo, envolvendo o dente 38 (Figura 5). As hipóteses diagnósticas foram COS e tumor odontogênico.

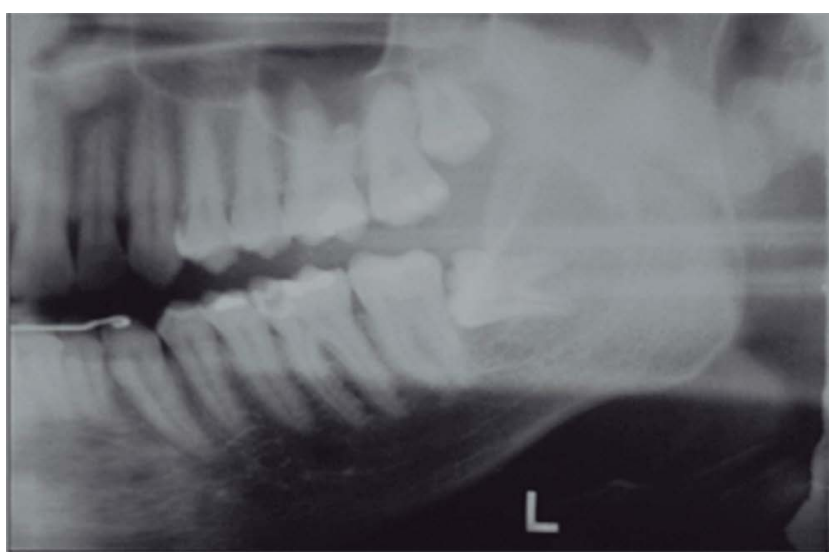

Fonte: dos autores.

Figura 5 - Radiografia panorâmica, evidenciando a lesão que envolve o dente 38

Como forma de tratamento optou-se por realizar a exodontia do elemento 38 e exploração cirúrgica da lesão. Durante a cirurgia, observou-se ausência de revestimento epitelial e a cavidade encontrava-se vazia, contendo não mais que um pequeno fluido serosanguinolento. Baseado nas características clínicas, radiográficas e transcirúrgicas, o diagnóstico foi de COS. Realizou-se, então, nesse momento, a curetagem da lesão. Após seis meses, foi realizada radiografia de controle, na qual foi possível observar reparo ósseo na região (Figura 6). 


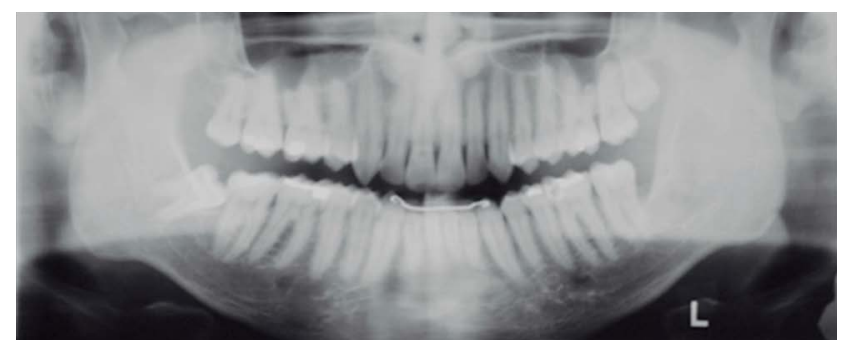

Fonte: dos autores.

Figura 6 - Radiografia panorâmica seis meses após a cirurgia

\section{Discussão}

O COS é uma lesão rara dos maxilares, incluída na classificação da OMS no grupo de lesões ósseas relacionadas, juntamente com o fibroma ossificante, as displasias fibrosa e óssea, a lesão central de células gigantes, o cisto ósseo aneurismático e o querubismo1. Embora tenha sido descrito primeiramente no início do século XX, a patogênese do COS permanece incerta e tem sido sujeita a muita especulação $0^{14-15}$. Trata-se de uma patologia de difícil diagnóstico, visto que existem inúmeras lesões com características clínicas e radiográficas semelhantes ao COS.

Os pacientes tinham entre 16 e 22 anos de idade, estando de acordo com a prevalência encontrada na literatura, que afirma tratar-se de lesão predominante em jovens na segunda década de vida ${ }^{5}$. Há uma concordância da literatura de que o COS apresenta-se assintomático, sendo assim, mais frequentemente diagnosticado durante radiografias para outros motivos, os dentes que parecem estar envolvidos na lesão são geralmente vitais, não mostram reabsorção radicular e não há evidencias de deslocamento dental ${ }^{7}$. Essas características puderam ser observadas nos dois primeiros casos relatados.

Radiograficamente, o COS geralmente aparece como uma área radiolúcida unilocular, na região posterior da mandíbula, com as margens festonadas entre as raízes dentárias ${ }^{16-17}$, característica encontrada no segundo caso clínico relatado. Esse aspecto, de projeções em forma de cúpula, também pode ser observado no primeiro caso de forma bem evidente (Figura 1), porém, quanto à localização, o COS encontrava-se em região mais anterior, entre o incisivo lateral e o segundo pré-molar, localização rara para esse tipo de lesão.

Variações nesse padrão radiográfico são comuns, como a presença de multiloculações associada com expansão cortical e aumento lento, lesões extensas, envolvendo uma porção substancial do corpo e ramo ascendente, associação com dentes inclusos/ impactados e múltiplas ocorrências em um mesmo paciente $^{18}$. O terceiro caso apresenta algumas dessas variações, uma vez que o COS encontra-se em região de ângulo da mandíbula e está associado a um dente incluso (Figura 5). Dessa forma, o COS deve ser incluído no diagnóstico diferencial das lesões radiolúcidas dos maxilares, incluindo o cisto dentígero, o tumor odontogênico queratocístico, ameloblastoma, o mixoma odontogênico, o cisto ósseo aneurismático, o defeito osteoporótico focal da medula, as más formações vasculares intraósseas e a lesão central de células gigantes, entre outros.

Cistos simples similares podem estar associados a lesões de displasia cemento-óssea florida (DCOF) e outras proliferações fibro-ósseas. Isso ocorre tipicamente em pacientes idosos ${ }^{19}$. De acordo com Wakasa et $a^{20}{ }^{20}(2002)$, a DCOF pode preceder o COS nos casos de associação entre as lesões, sugerindo que a produção desordenada de trabéculas na DCOF possa resultar na obstrução da drenagem linfática, induzindo à formação do COS. No entanto, nos casos clínicos relatados não havia essa associação.

A forma de tratamento do COS, no primeiro e no terceiro caso, foi a cirurgia por meio de curetagem rigorosa para estimular o sangramento dentro da cavidade que se encontra vazia, tratamento esse, acordado por diversos autores na literatura ${ }^{9-11}$. Por apresentar características clínicas e radiográficas bastante sugestivas de COS, no segundo caso, pode-se optar somente pela proservação, uma vez que a resolução espontânea também é relatada na literatura ${ }^{13}$. Todos os casos evoluíram de maneira satisfatória.

\section{Considerações finais}

O COS ainda é uma lesão com etiopatogênia indefinida, que pode atingir pacientes principalmente durante a segunda década de vida. Trata-se de uma lesão assintomática, os dentes que parecem estar envolvidos na lesão são geralmente vitais, não mostram reabsorção radicular e não há evidências de deslocamento dental. Como frequentemente é obtido pouco ou nenhum tecido no momento da cirurgia, o diagnóstico do COS é baseado primariamente em características clínicas e radiográficas juntamente com os achados cirúrgicos. Radiograficamente, apresenta-se como uma lesão radiolúcida, bem delimitada. Podem ser observadas interdigitações entre as raízes dentárias. A forma de tratamento indicada é a curetagem da lesão, com acompanhamento radiográfico pós-cirúrgico para proservação do caso, ou nos casos em que as características clínicas e radiográficas são bastante sugestivas, pode-se optar somente pela proservação.

\section{Abstract}

Introduction: the Simple Bone Cyst (SBC) is classified by the World Health Organization (WHO) as a non-neoplastic lesion related to bones, defined as an intraosseous cyst. The cause and pathogenesis are uncertain and controversial. Most injuries are empty cavities containing no more than a small serous or serosanguineous fluid, with absent epithelial lining. The most affected areas are long and gnathic bones. Among the gnathic bones the mandible is more frequently affected, mainly 
in the molar and premolar regions. It has an asymptomatic nature, but pain and paresthesia may be reported in some cases. Objective: this paper aims to report three cases of SBC with radiographic manifestations and different therapeutic approaches. Final considerations: the diagnosis of $S B C$ is primarily based on clinical and radiographic features along with surgical findings. Radiographically, it appears as a radiolucent and well-defined lesion, and scalloping may be observed among dental roots. Lesion curettage is the indicated treatment, with postoperative radiographic follow-up for proservation of the case, or in cases where the clinical and radiographic features are quite suggestive, proservation alone may be advised.

Keywords: Bone cysts. Mandible. Panoramic radiography.

\section{Referências}

1. World health organization classification of tumours (WHO) - International Agency for Research on Cancer (IARC), IARCPress Lyon, 2005.

2. Tong ACK, Ng IOL, Yan BSW. Variations in clinical presentations of the simple bone cyst: report of cases. J Oral Maxillofac Surg. 2003;(61):1487-91.

3. MacDonald-Jankowski DS. Traumatic bone cysts in the jaws of a Hong Kong Chinese population. Clinical Radiol. 1995;(50):787-97.

4. Bensahel, H., Jehanno, P. Solitary bone cyst: controversies and treatment. J. PediatrOrthop 1998;(7):257-61.

5. Neville BW, Damm DD, Allem CM, Bouquot JE. Patologia Oral e Maxilofacial, 3. ed. Rio de Janeiro: Elsevier; 2009.

6. Copete MA, Kawamata A, Langlais RP. Solitary bone cyst of the jaws: radiographic review of 44 cases. Oral Surg Oral Med Oral Pathol Oral RadiolEndod. 1998;85(2):221-5.

7. Harris SJ, O Carroll MK, Gordy FM. Idiopathic bone cavity (traumatic bonecyst) with the radiographic appearance of a fibro-osseous lesion. Oral SurgOralMed Oral Pathol. 1992;74(1):118-23.

8. Bezerra JRS, Silva ARS, Jorge Jr. J, Gouvêa AF, Lopes MA. Atypical presentations of simple bone cysts of the mandible: A case series and review of literature. J CranioMaxilloFac Surg. 2013;41:391-6.

9. Baqain ZH, Jayakrishnan A, Farthing PM, Hardee P. Recurrence of a solitary bone cyst of the mandible: case report. $\mathrm{Br}$ J Oral Maxillofac Surg. 2005;43:333-5.

10. Lago CA, Cauás M, Pereira AM, Portela L. Cisto ósseo traumático em mandíbula: relato de caso. RevCirTraumatol Buco-Maxilo-Fac. 2005;6:23-8.

11. Diago MP, Sanchis-Bielsa JM, Marco JB, Minguez-Sanz JM. Surgical treatment and follow-up of solitary bone cyst of the mandible: a report of seven cases. Br J Oral Maxillofac Surg. 2001;39:221-3.

12. Sverzut CE. Cisto Ósseo Solitário: relato de um caso clínico. Rev. Dental Press OrtodonOrtop Facial. 2002;7:63-7.

13. PaesBLl,Santos KCP,Marcucci M, Claudio Costa,Oliveira JX. Cisto ósseo simples: avaliação radiográfica, anatomopatológica e clínica de seis casos. J Health Sci Inst. 2010;28(1):71-6.

14. Sapp JP, Stark ML. Self-healing traumatic bone cysts. Oral SurgOral Med Oral Pathol. 1990;69(5):597-602.
15. Tong AC, Ng IO, Yan BS. Variations in clinical presentations of the simple bone cyst: report of cases. J Oral Maxillofac Surg. 2003;61(12):1487-91.

16. Howe GL. "Haemorrhagic cysts" of the mandible. I. Br J Oral Surg. 1965;3(1):55-76.

17. Harnet JC, Lombardi T, Klewansky P, Rieger J, Tempe $\mathrm{MH}$, Clavert JM. Solitary bone cyst of the jaws: a review of the etiopathogenic hypotheses. J Oral Maxillofac Surg. 2008;66(11):2345-8.

18. Forssell K, Forssell H, Happonen RP, Neva M. Simple bone cyst: review of the literature and analysis of 23 cases. Int $J$ Oral Maxillofac Surg. 1988;17(1):21-4.

19. Kuhmichel A, Bouloux GF. Multifocal traumatic bone cysts: case report and current thoughts on etiology. J Oral Maxillofac Surg. 2010;68(1):208-12.

20. Wakasa T, Kawai N, Aiga H, Kishi K. Management of florid cemento-osseous dysplasia of the mandible producing solitary bone cyst: report of a case. J. Oral Maxillofac Surg. 2002;60(7):832-5.

\section{Endereço para correspondência:}

Flávia Carneiro Tagliari Bisol

Paranaguá, $\mathrm{n}^{\circ} 346$, zona 7

87020-190 Maringá-PR

Fone: (44) 99597959

E-mail: fla.tagliari@hotmail.com

Recebido: 29/07/2014. Aceito: 15/12/2014. 